\title{
Chemical reaction and radiation absorption effects on the flow and heat transfer of a nanofluid in a rotating system
}

\author{
B. Venkateswarlu $\cdot$ P. V. Satya Narayana
}

Received: 11 May 2014/ Accepted: 24 May 2014/Published online: 22 June 2014

(C) The Author(s) 2014. This article is published with open access at Springerlink.com

\begin{abstract}
The aim of this paper is to study the effects of radiation absorption and chemical reaction on MHD free convection heat transfer flow of a nanofluid bounded by a semi-infinite flat plate in a rotating frame of reference. The plate is assumed to oscillate in time with steady frequency so that the solutions of the boundary layer are the similar oscillatory kind. The entire system rotates about the axes normal to the plate. The dimensionless governing differential equations for this investigation are solved analytically using perturbation method. The effects of various important parameters entering into the problem on velocity, temperature, skin friction and Nusselt number within the boundary layer are discussed for $\mathrm{Cu}$-water-based nanofluid with the help of graphs. The predicted consequences obviously point out that the presence of nanoparticles in the base fluid improves the heat transfer process significantly. The results also show that the values of Nusselt number in case of nanofluid are more pronounced than that of micropolar fluid.
\end{abstract}

Keywords Nanofluid - Chemical reaction - Thermal radiation $\cdot$ Rotating frame $\cdot$ Radiation absorption $\cdot$ MHD

B. Venkateswarlu

Department of Mathematics, Sree Vidyanikethan Engineering

College, A. Rangampet, Tirupati, A.P, India

e-mail: bvenkateswarlu.maths@gamil.com

P. V. Satya Narayana $(\bowtie)$

Fluid Dynamics Division, SAS, VIT University,

Vellore 632 014, T.N, India

e-mail: pvsatya8@yahoo.co.in

\section{Introduction}

Nanofluid is a term initially used by Choi (1995) and refers to a new class of heat transfer fluids with superior thermal properties. It contains a base fluid and nanometer-sized (between 1 and $100 \mathrm{~nm}$ in diameter) particles or fibers suspended in ordinary fluid, called nanoparticles. The suspended ultra-fine particles change transport properties and heat transfer performance of the nanofluid. The study of heat transfer in the presence of nanofluids is of great practical importance to engineers and scientists because of their universal occurrence in many branches of science and engineering, viz. cooling applications of nanofluids, medical applications (cancer therapy and safer surgery) and other possible areas (electronic devices for use in military systems, airplanes).

Theoretical and experimental investigations have been conducted to measure the improvement in the thermal conductivity of nanofluids [Bachok et al. (2010a); Khanafer et al. (2003) and Makinde and Aziz (2011)]. Experimental studies in literature [Yu et al. (2008) and Kakac and Pramuanjaroenkij (2009)] show that the typical thermal conductivity enhancements are in the range $15-40 \%$ over the base fluid and heat transfer coefficient enhancements have been found up to $40 \%$. Choi et al. (2001) observed that the thermal conductivity increases up to two times when small amount (less than $1 \%$ by volume) of nanoparticles is added to the ordinary fluids. There is a vast amount of literature on the flow of nanofluid model proposed by Buongiorno (2006), but we are referring a few recent studies [Rana and Bharava (2012); Alsaedi and Hayat (2012) and Mastaneh and Asghar (2012)]. However, we are following the nanofluid model proposed by Tiwari and Das (2007), which is being used by many current researchers Abu-Nada (2008); (Abu-Nada and Oztop 
2009); Muthtamilselvan et al. (2010) and Ahmad et al. (2011) on different flow fields.

The study of heat transfer for an electrically conducting fluid past a porous plate under the influence of a magnetic field in a rotating frame of reference has attracted the interest of many investigators in view of its applications in many industrial (in the design of turbines and turbo mechanics), astrophysical (dealing with the sunspot development, the solar cycle and the structure of rotating magnetic stars), geophysical (hydrologists to study the migration of underground water, petroleum engineers to observe the movement of oil and gas through the reservoir), technological and engineering applications (MHD generators, ion propulsion, MHD pumps, etc.) and many other practical applications, that is, in biomechanical problems (e.g., blood, flow in the pulmonary alveolar sheet). The pioneering works of Bachok et al. (2010b) and (2011) have laid the foundation stone in this field. Also many authors have studied the flow and heat transfer in rotating system with various geometrical situations [Mazumder (1991) and Satya Narayana et al. (2013c)]. Nield and Kuznetsov (2009) presented an analytical study for natural convection past a vertical plate in a porous medium saturated by a nanofluid. Recently, Uddin and Harmand (2013) have investigated the influence of natural convection heat transfer effects of nanofluids along a vertical plate in porous medium. Chamkha et al. (2011) have analyzed the natural convection past a sphere embedded in a porous medium saturated by a nanofluid.

Many processes in engineering areas occur at high temperature and knowledge of radiation heat transfer becomes vital for the design of the pertinent equipment such as the sensible heat storage bed, the nuclear power plants, the nuclear reactor cooling system, gas turbines and the various propulsion devices, aircrafts, missiles, satellites and space vehicles and underground nuclear waste disposal, etc. Chamkha et al. (2012) studied the radiation effects on mixed convection over wedge with a nanofluid. They found that the effects of the radiation conduction parameter and the surface temperature parameter are significantly stronger on the local Nusselt number than that of the local Sherwood number. Recently, Satya Narayana et al. (2013b) have studied the influence of thermal radiation and heat source on MHD nanofluid past a vertical plate in a rotating system. More recently, Liancun Zheng et al. (2013) studied the flow and radiation heat transfer of a nanofluid over a stretching sheet with velocity slip in porous medium. The effects of thermal radiation were also investigated by many researchers Hady et al. (2012) and Olanrewaju et al. (2012).

The study of heat transfer with chemical reaction in the presence nanofluids is of immense realistic significance to engineers and scientists, because of its almost universal incidence in many branches of science and engineering. This phenomenon plays an important role in chemical industry, power and cooling industry for drying, evaporation, energy transfer in a cooling tower and the flow in a desert cooler, chemical vapor deposition on surfaces, cooling of nuclear reactors and petroleum industries and also there are many transport processes that are governed by the joint action of the buoyancy forces from both thermal and mass diffusion in the presence of chemical reaction effects. The effect of chemical reaction with heat radiation in presence of nanofluid over a porous vertical stretching surface is investigated by Rosmila Abdul-Kahar et al. (2011). Kameswaran et al. (2012) have analyzed the hydromagnetic nanofluid flow due to a stretching or shrinking sheet with viscous dissipation and chemical reaction effects. Recently, many researchers (Ellahi 2013; Ellahi et al. 2013; Narahari et al. 2013; Umavathi 2013) have studied the flow and heat transfer characteristic of Newtonian/non-Newtonian nanofluids over various geometries.

To the author's knowledge, no studies have this far been communicated with regard to study the flow and heat transfer distinctiveness of a nanofluid past a vertical flat plate with chemical reaction, radiation absorption and thermal radiation in a rotating frame of reference. So the main aim of this study is to extend the effort of reference Satya Narayana et al. (2013b) in two directions: (1) to consider radiation absorption (2) to consider the mass transfer effects. Analytical closed form solutions are obtained for the momentum and the energy equations using the regular perturbation method. Graphs are used to illustrate the significance of key parameters on the nanofluid velocity and temperature distributions.

\section{Mathematical formulation of the problem}

We consider an unsteady three-dimensional flow of an electrically conducting incompressible nanofluid past a semi-infinite vertical permeable plate with chemical reaction and radiation absorption. A uniform external magnetic field $B_{0}$ is taken to be acting along the $\bar{z}$ axis. It is assumed that there is no applied voltage which implies the absence of an electric field. The flow is assumed to be in the $\bar{x}$-direction which is taken along the plate in the upward direction and $\bar{z}$ axis is normal to the plate (see Fig. 1). Also it is assumed the whole system is rotated with a constant vector $\Omega$ about $\bar{z}$-axis. The fluid is assumed to be gray, absorbing emitting but not scattering medium. The radiation heat flux in $\bar{x}$-direction is considered negligible in comparison that in the $\bar{z}$-direction. Due to semi-infinite plate surface assumption the flow variables are functions of $z$ and time $t$ only. 
The governing equations for this investigation are given by:

$$
\begin{aligned}
& \frac{\partial \bar{w}}{\partial \bar{z}}=0 \\
& \frac{\partial \bar{u}}{\partial \bar{t}}+\bar{w} \frac{\partial \bar{u}}{\partial \bar{z}}-2 \Omega \bar{v}=\frac{1}{\rho_{\mathrm{nf}}}\left\{\mu_{\mathrm{nf}} \frac{\partial^{2} \bar{u}}{\partial \bar{z}^{2}}+(\rho \beta)_{\mathrm{nf}} g\left(T-T_{\infty}\right)\right. \\
& \left.-\frac{v_{f} \bar{u}}{k}-\sigma B_{o}^{2} \bar{u}\right\} \\
& \frac{\partial \bar{v}}{\partial \bar{t}}+\bar{w} \frac{\partial \bar{v}}{\partial \bar{z}}+2 \Omega \bar{u}=\frac{1}{\rho_{\mathrm{nf}}}\left\{\mu_{\mathrm{nf}} \frac{\partial^{2} \bar{v}}{\partial \bar{z}^{2}}-\frac{v_{f} \bar{u}}{k}-\sigma B_{o}^{2} \bar{v}\right\} \\
& \frac{\partial T}{\partial \bar{t}}+\bar{w} \frac{\partial T}{\partial \bar{z}}=\alpha_{\mathrm{nf}} \frac{\partial^{2} T}{\partial \bar{z}^{2}}-\frac{1}{\left(\rho C_{P}\right)_{\mathrm{nf}}} \frac{\partial q_{r}}{\partial \bar{z}}-\frac{Q^{\prime}}{\left(\rho C_{p}\right)_{\mathrm{nf}}}\left(T-T_{\infty}\right) \\
& +Q_{l}^{\prime}\left(C-C_{\infty}\right) \\
& \frac{\partial C}{\partial \bar{t}}+\bar{w} \frac{\partial C}{\partial \bar{z}}=D_{B} \frac{\partial^{2} C}{\partial \bar{z}^{2}}-K_{l}\left(C-C_{\infty}\right)
\end{aligned}
$$
by

The boundary conditions for the problem are given $\bar{u}(\bar{z}, \bar{t})=0 \quad \bar{v}(\bar{z}, \bar{t})=0 \quad T=T_{\infty}$ $C=C_{\infty} \quad$ for $\bar{t} \leq 0$ and any $\bar{z}$ $\bar{u}(0, \vec{t})=U_{0}\left\{1+\frac{\varepsilon}{2}\left(e^{\mathrm{int}}+e^{-\mathrm{int}}\right)\right\} \quad \bar{v}(0, \vec{t})=0$

$T(0, \bar{t})=T_{\infty}$

$\bar{u}(\infty, \bar{t}) \rightarrow 0 \quad \bar{v}(\infty, \bar{t}) \rightarrow 0 \quad T(\infty, \bar{t}) \rightarrow T_{\infty}$

$C(\infty, \bar{t}) \rightarrow C_{\infty}$ for $\bar{t} \geq 0$

The properties of nanofluids are given as follows

$$
\begin{aligned}
\rho_{\mathrm{nf}}= & (1-\phi) \rho_{f}+\phi \rho_{s} \quad\left(\rho C_{p}\right)_{\mathrm{nf}}=(1-\phi)\left(\rho C_{p}\right)_{f} \\
& +\phi\left(\rho C_{p}\right)_{s} \quad(\rho \beta)_{\mathrm{nf}}=(1-\phi)(\rho \beta)_{f}+\phi(\rho \beta)_{s} \\
\mu_{\mathrm{nf}}= & \frac{\mu_{f}}{(1-\phi)^{2.5}} \quad \alpha_{\mathrm{nf}}=\frac{K_{\mathrm{nf}}}{\left(\rho C_{p}\right)_{\mathrm{nf}}} \\
K_{\mathrm{nf}}= & K_{f}\left\{\frac{K_{s}+2 K_{f}-2 \phi\left(K_{f}-K_{s}\right)}{K_{s}+2 K_{f}+2 \phi\left(K_{f}-K_{s}\right)}\right\}
\end{aligned}
$$

We consider the solution of Eq. (1) as

$$
\bar{w}=-w_{0}
$$

The radiative heat term (Brewster 1972) by using the Rosseland approximation is given by:

$$
\begin{aligned}
& q_{r}=\frac{-4 \sigma^{*}}{3 k^{*}} \frac{\partial T^{4}}{\partial z} \\
& T^{4}=4 T_{\infty}^{3} T-3 T_{\infty}^{4} \\
& \frac{\partial q_{r}}{\partial \bar{z}}=-16 \frac{T_{\infty}^{3} \sigma^{*}}{3 k^{*}} \frac{\partial^{2} T}{\partial \bar{z}^{2}} .
\end{aligned}
$$

We introduce the following dimensionless variables

$$
\begin{aligned}
& z=\left(\frac{U_{0}}{v_{f}}\right) \bar{z} \quad t=\left(\frac{U_{0}^{2}}{v_{f}}\right) \bar{t} \quad n=\left(v_{f} / U_{0}^{2}\right) \bar{n} \\
& u=\frac{\bar{u}}{U_{0}} \quad v=\frac{\bar{v}}{U_{0}} \quad K=\frac{k \rho_{f} u_{0}^{2}}{v_{f}^{2}} \\
& S=\frac{w_{0}}{U_{0}} \quad M=\frac{\sigma B_{0}^{2} v_{f}}{\rho_{f} U_{o}^{2}} \quad R=\frac{2 \Omega v_{f}}{U_{0}^{2}} \\
& Q_{H}=\frac{Q^{\prime} v_{f}^{2}}{K_{f} U_{0}^{2}} \quad \operatorname{Pr}=\frac{v_{f}\left(\rho C_{p}\right)_{f}}{K_{f}} \\
& K r=\frac{K_{l} V_{f}}{U_{0}^{2}} \quad F=\frac{4 \sigma^{*} T_{\infty}^{3}}{k k^{*}} \quad \theta=\frac{T-T_{\infty}}{T_{w}-T_{\infty}} \\
& \psi=\frac{C-C_{\infty}}{C_{w}-C_{\infty}} \quad Q_{l}=\frac{Q_{l}^{\prime}\left(C_{w}-C_{\infty}\right)}{U_{0}^{2}\left(T_{w}-T_{\infty}\right)}
\end{aligned}
$$

Substituting Eq. (12) into Eqs. (2)-(5) yields the following dimensionless equations (dropping primes):

$\frac{\partial u}{\partial t}-S \frac{\partial u}{\partial z}-R v=\frac{1}{A_{1} A_{3}} \frac{\partial^{2} u}{\partial z^{2}}+\frac{A_{4}}{A_{3}} \theta-\frac{1}{A_{3}}\{M+1 / K\} u$

$\frac{\partial v}{\partial t}-S \frac{\partial v}{\partial z}+R u=\frac{1}{A_{1} A_{3}} \frac{\partial^{2} v}{\partial z^{2}}-\frac{1}{A_{3}}\{M+1 / K\} v$

$\frac{\partial \theta}{\partial t}-S \frac{\partial \theta}{\partial z}=\frac{1}{\operatorname{Pr}}\left\{\frac{A_{2}}{A_{5}} \frac{\partial^{2} \theta}{\partial z^{2}}-\frac{Q_{H}}{A_{5}} \theta\right\}+Q_{l} \psi$

$\frac{\partial \psi}{\partial t}-S \frac{\partial \psi}{\partial z}=\frac{1}{S c} \frac{\partial^{2} \psi}{\partial z^{2}}-K r \psi$

The boundary condition (6) becomes

$$
\begin{aligned}
& u(z, t)=0, \quad v(z, t)=0, \quad \theta(z, t)=0, \\
& \psi(z, t)=0 \quad \text { for } t \leq 0 \text { and any } z \\
& u(0, t)=1+\frac{\varepsilon}{2}\left\{e^{\text {int }}+e^{- \text {int }}\right\}, \quad v(0, t)=0, \\
& \theta(0, t)=1, \quad \psi(0, t)=1 \\
& u(\infty, t) \rightarrow 0, \quad v(\infty, t) \rightarrow 0, \quad \theta(\infty, t) \rightarrow 0, \\
& \psi(\infty, t) \rightarrow 0 \quad \text { for } t \geq 0
\end{aligned}
$$

Using Eq. (13) the velocity characteristic $U_{0}$ is defined as: $U_{0}=\left\{g \beta_{f}\left(T_{w}-T_{\infty}\right) v_{f}\right\}^{1 / 3}$

We now simplify Eqs. (13)-(16) by putting the fluid velocity in the complex form as

$\chi(z, t)=u(z, t)+i v(z, t)$

By using (18) we can simplify Eqs. (13) and (14) to the following equation

$\frac{\partial \chi}{\partial t}-S \frac{\partial \chi}{\partial z}+i R \chi=\frac{1}{A_{1} A_{3}} \frac{\partial^{2} \chi}{\partial z^{2}}+\frac{A_{4}}{A_{3}} \theta-\frac{1}{A_{3}}\{M+1 / K\} \chi$ 
The boundary conditions (17) become

$$
\begin{aligned}
& \chi(z, t)=0, \quad \theta(z, t)=0, \quad \psi(z, t)=0 \\
& \quad \text { for } t \leq 0 \text { and any } z \\
& \chi(0, t)=1+\frac{\varepsilon}{2}\left\{e^{\text {int }}+e^{- \text {int }}\right\}, \quad \theta(0, t)=1, \\
& \quad \psi(0, t)=1 \\
& \chi(\infty, t) \rightarrow 0, \quad \theta(\infty, t) \rightarrow 0, \quad \psi(\infty, t) \rightarrow 0 \\
& \quad \text { for } t \geq 0
\end{aligned}
$$

Analytical solutions

In order to solve the above system of partial differential Eqs. (13)-(16) under the boundary conditions (17) in the neighborhood of the plate, we assume that [see Ganapathy (1994)]

$$
\begin{aligned}
& \chi(z, t)=\chi_{0}(z, t)+\frac{\varepsilon}{2}\left\{e^{\mathrm{int}} \chi_{1}+e^{-\mathrm{int}} \chi_{2}\right\} \\
& \theta(z, t)=\theta_{0}(z, t)+\frac{\varepsilon}{2}\left\{e^{\mathrm{int}} \theta_{1}+e^{-\mathrm{int}} \theta_{2}\right\} \\
& \psi(z, t)=\psi_{0}(z, t)+\frac{\varepsilon}{2}\left\{e^{\mathrm{int}} \psi_{1}+e^{-\mathrm{int}} \psi_{2}\right\}
\end{aligned}
$$

Invoking the above Eqs. (21)-(23) into the Eqs. (13)(16) and equating the harmonic and non-harmonic terms and neglecting the higher order terms of $O\left(\varepsilon^{2}\right)$, we obtain the following set of equations.

Zeroth order equations are

$\chi_{0}^{\prime \prime}+A_{3} S \chi_{0}^{\prime}-A_{1}\left\{i R A_{3}+M+1 / K\right\} \chi_{0}+A_{1} A_{4} \theta_{0}=0$

$A_{2} \theta_{0}^{\prime \prime}+A_{5} S \operatorname{Pr} \theta_{0}^{\prime}-Q_{H} \theta_{0}+A_{5} \operatorname{Pr} Q_{l} \psi_{0}=0$

$\psi_{0}^{\prime \prime}+S S c \psi_{0}^{\prime}-\operatorname{Kr} S c \psi_{0}=0$

First order equations are:

$\chi_{1}^{\prime \prime}+A_{3} S \chi_{1}^{\prime}-A_{1}\left\{i(R+n) A_{3}+M+1 / K\right\} \chi_{1}+A_{1} A_{4} \theta_{1}=0$

$A_{2} \theta_{1}^{\prime \prime}+A_{5} S \operatorname{Pr} \theta_{1}^{\prime}-\left\{i n A_{5} \operatorname{Pr}+Q_{H}\right\} \theta_{1}+A_{5} \operatorname{Pr} Q_{l} \psi_{1}=0$

$\psi_{1}^{\prime \prime}+S S c \psi_{1}^{\prime}-S c($ in $+K r) \psi_{1}=0$.

Second order equations are:

$\chi_{2}^{\prime \prime}+A_{3} S \chi_{2}^{\prime}-A_{1}\left\{i(R-n) A_{3}+M+1 / K\right\} \chi_{2}+A_{1} A_{4} \theta_{2}=0$

$A_{2} \theta_{2}^{\prime \prime}+A_{5} S \operatorname{Pr} \theta_{2}^{\prime}-\left\{i n A_{5} \operatorname{Pr}-Q_{H}\right\} \theta_{2}+A_{5} \operatorname{Pr} Q_{l} \psi_{2}=0$

$\psi_{2}^{\prime \prime}+S S c \psi_{2}^{\prime}-S c($ in $-K r) \psi_{2}=0$,

where primes denote differentiation with respect to $z$.
The corresponding boundary conditions can be written as:

$\chi_{0}=1, \quad \theta_{0}=1, \quad \psi_{0}=1 \chi_{1}=1, \theta_{1}=0, \quad \psi_{1}=0$,

$\chi_{2}=1, \theta_{2}=0, \quad \psi_{2}=0 \quad$ at $z=0$

$\chi_{0} \rightarrow 0, \theta_{0} \rightarrow 0, \psi_{0} \rightarrow 0, \chi_{1} \rightarrow 0, \theta_{1} \rightarrow 0$,

$\psi_{1} \rightarrow 0 \quad \chi_{2} \rightarrow 0, \theta_{2} \rightarrow 0, \psi_{2} \rightarrow 0$ at $z \rightarrow 0$

Solving Eqs. (24)-(32) with the boundary conditions (33), we obtain the expression for velocity, temperature and concentration as:

$$
\begin{aligned}
\chi(z, t)= & B_{3} e^{-m_{1} z}+B_{4} e^{-m_{2} z}+B_{5} e^{-m_{3} z} \\
& +\frac{\varepsilon}{2}\left\{e^{-m_{4} z} e^{\text {int }}+e^{-m_{5} z} e^{-\mathrm{int}}\right\} \\
\theta(z, t)= & B_{1} e^{-m_{1} z}+B_{2} e^{-m_{2} z} \\
\psi(z, t)= & e^{-m_{1} z}
\end{aligned}
$$

The physical quantities of engineering interest are skinfriction coefficient, Nusselt number and Sherwood number.

The local skin-friction coefficient $C_{f}$ is given by:

$$
\begin{aligned}
C_{f} & =\frac{\bar{\tau}_{w}}{\rho_{f} U_{0}^{2}} \Rightarrow \frac{1}{(1-\phi)^{2.5}} \chi^{\prime}(0) \\
& =\frac{-1}{A_{1}}\left\{m_{1} B_{3}+m_{2} B_{4}+m_{3} B_{5}+\frac{\varepsilon}{2}\left\{m_{4} e^{\text {int }}+m_{5} e^{-\mathrm{int}}\right\}\right\}
\end{aligned}
$$

The local Nusselt number $N u$ is given by

$\mathrm{Nu}=\frac{\bar{x} \bar{q}_{w}}{k_{f}\left(T_{w}-T_{\infty}\right)} \Rightarrow \frac{-k_{\mathrm{nf}}}{k_{f}} \theta^{\prime}(0)=\frac{k_{\mathrm{nf}}}{k_{f}}\left\{m_{1} B_{1}+m_{2} B_{2}\right\}$

The local Sherwood number $\mathrm{Sh}_{x}$ is given by:

$\mathrm{Sh}_{x}=\frac{\bar{x} \bar{q}_{m}}{D_{B}\left(C_{w}-C_{\infty}\right)} \Rightarrow-x \psi^{\prime}(0)=m_{1}$

where $\bar{\tau}, \bar{q}_{w}$ and $\bar{q}_{m}$ are the wall shear stress or skin friction, the wall heat flux and the wall mass flux from the plate, respectively, which are given by:

$$
\begin{aligned}
\bar{\tau} & =\mu_{\mathrm{nf}}\left(\frac{\partial \bar{u}}{\partial \bar{z}}\right)_{\bar{z}=0} \quad \bar{q}_{w}=-k_{\mathrm{nf}}\left(\frac{\partial T}{\partial \bar{z}}\right)_{\bar{z}=0} \quad \bar{q}_{m} \\
& =-\mu_{\mathrm{nf}}\left(\frac{\partial C}{\partial \bar{z}}\right)_{\bar{z}=0} .
\end{aligned}
$$

\section{Results and discussion}

The formulation of the effect of chemical reaction and radiation absorption on MHD free convection heat and mass transfer flow of a nanofluid along a semi-infinite vertical plate in the presence of heat generation and a rotating frame of reference has been performed in the 
preceding sections. In order to get a physical insight into the flow problem, analytic solutions are conducted for various values of the parameters that describe the flow characteristics and the results are illustrated graphically in Figs. 2, 3, 4, 5, 6, 7, 8, 9, 10, 11, 12. They explain the fascinating features of important parameters on the nanofluid velocity, temperature, skin friction and Nusselt number distributions in a rotating system. We consider only copper $(\mathrm{Cu})$ water nanofluid with water as the base fluid. Table 1 shows the thermo-physical properties of water and the elements $\mathrm{Cu}, \mathrm{TiO}_{2}$ and $\mathrm{Al}_{2} \mathrm{O}_{3}$. In the present study we have chosen $n=10, \quad \mathrm{nt}=\pi / 2, \quad \operatorname{Pr}=6.2$, $\varepsilon=0.02$, while $\mathrm{Kr}, F, R, M, S, Q_{H}, Q_{1}$, Pr and Sc are varied over a range, which are listed in the figures legends. A comparison of the present results of skin-friction coefficient and rate of heat transfer with those of Satya Narayana et al. (2013b) and Hamad and Pop (2011) is provided in Table 2 for different values of Prandtl number. This table clearly displays that tremendous conformity exists between the results. This constructive judgment leads to assurance in the next section.

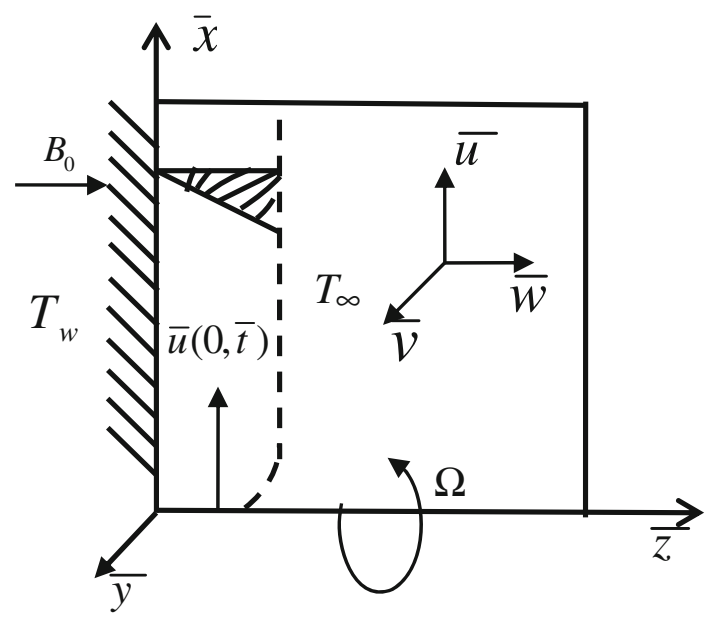

Fig. 1 Physical model and coordinate system of the problem

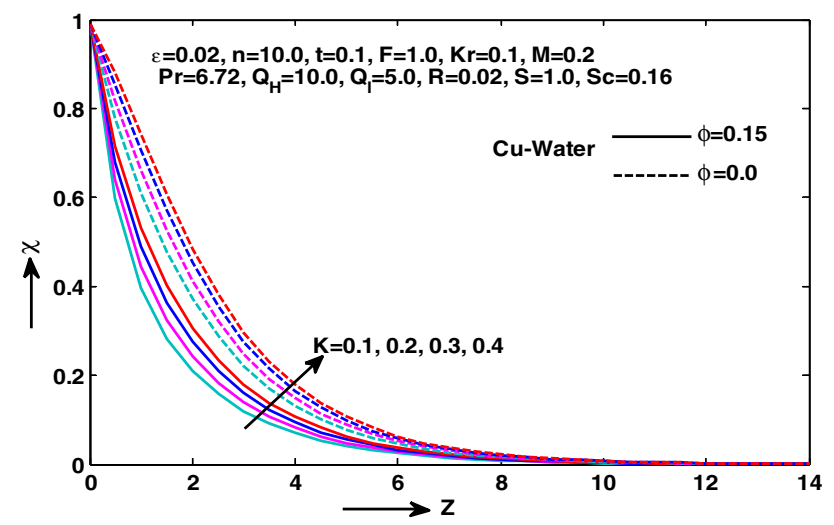

Fig. 2 Velocity for various values of $K$
Figure 2 illustrates the effect of permeability parameter $K$ on the nanofluid velocity distribution. It is clear from the figure that as $K$ increases, the nanofluid velocity also increases. Physically, this means that the porous medium impact on the boundary layer growth is significant due to the increase in the thickness of the thermal boundary layer. It is expected that, an increase in the permeability of the porous medium leads to the rise in the flow of fluid through it. When the holes of the porous medium become large, the resistance of the medium may be neglected. Further, we observe that the base fluid (pure water) exhibits relatively less velocity than that of the $\mathrm{Cu}$-water nanofluid. It is found that in both $x$ - and $y$-directions, nanofluid has less friction with plate as compare to the Newtonian (base) fluid, due to viscosity effects.

Figure $3 a, b$, respectively, shows the effect of thermal radiation parameter $F$ on the nanofluid velocity and temperature profiles. It is clear that, as the thermal radiation parameter $F$ increases, the nanofluid velocity and temperature distributions across the boundary layer increases. The increase in radiation parameter means the release of heat energy from the flow region and so the fluid temperature increases as the thermal boundary layer thickness become

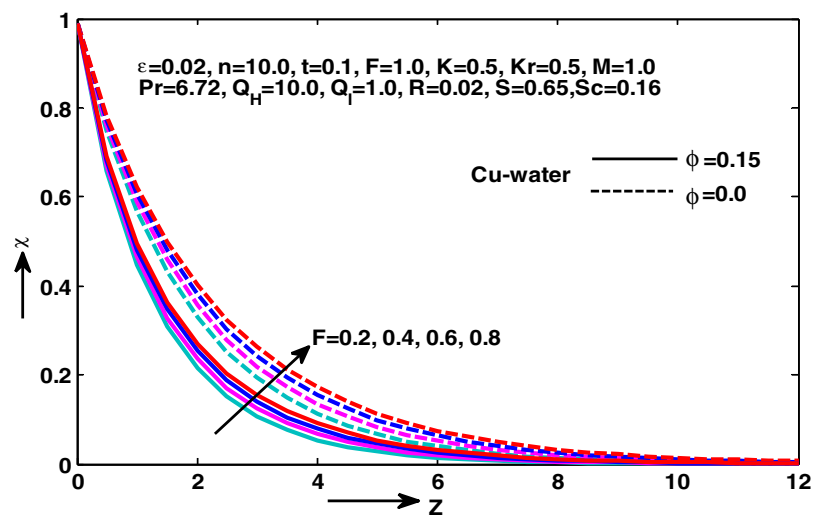

(a) Velocity for various values of $\mathrm{F}$

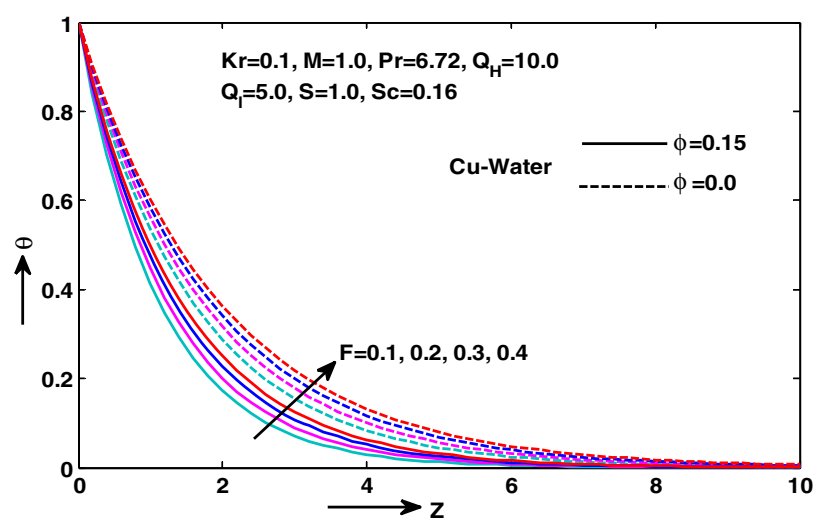

(b) Temperature for various values of $\mathrm{F}$

Fig. 3 a Velocity for various values of $F$. b Temperature for various values of $F$ 


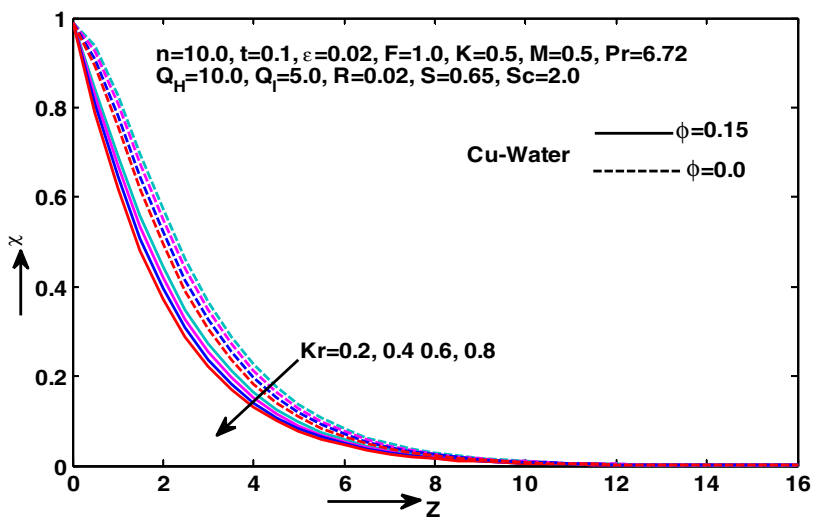

(a) Velocity for various values of $\mathrm{Kr}$

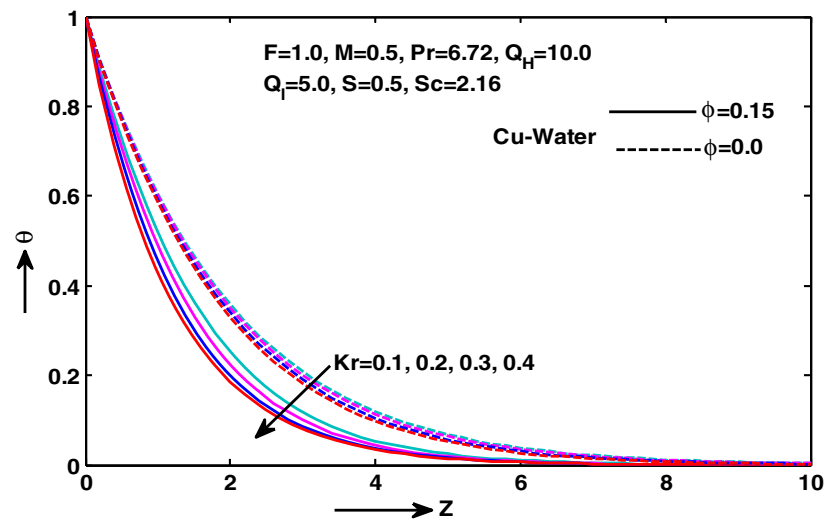

(b) Temperature for various values of $\mathrm{Kr}$

Fig. 4 a Velocity for various values of Kr. b Temperature for various values of $\mathrm{Kr}$

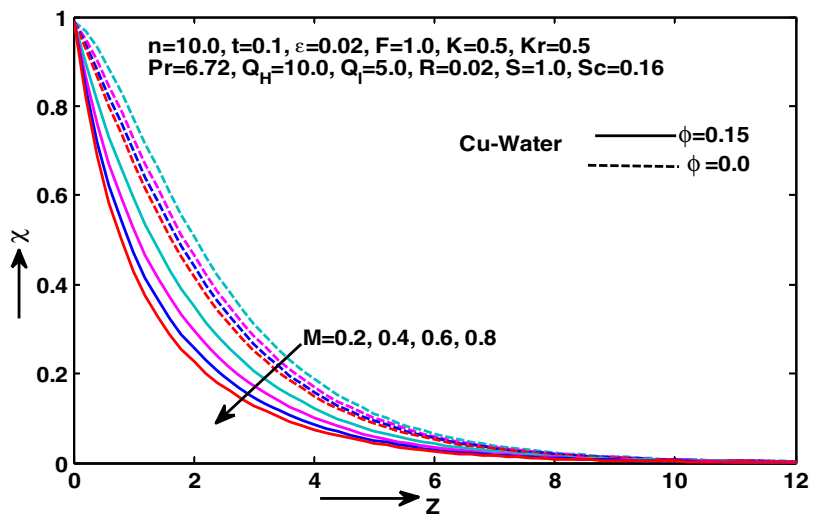

Fig. 5 Velocity for various values of $M$

thinner. In view of this explanation the effect of radiation becomes more significant as $F \rightarrow 0(F \neq 0)$ and can be neglected when $F \rightarrow \infty$. These figures illustrate this agreement with the physical behavior. These results are related to those obtained by Satya Narayana et al. (2013a), (b) and Hamad and Pop (2011).

The influence of the chemical reaction parameter $\mathrm{Kr}$ on the nanofluid velocity and temperature profiles,

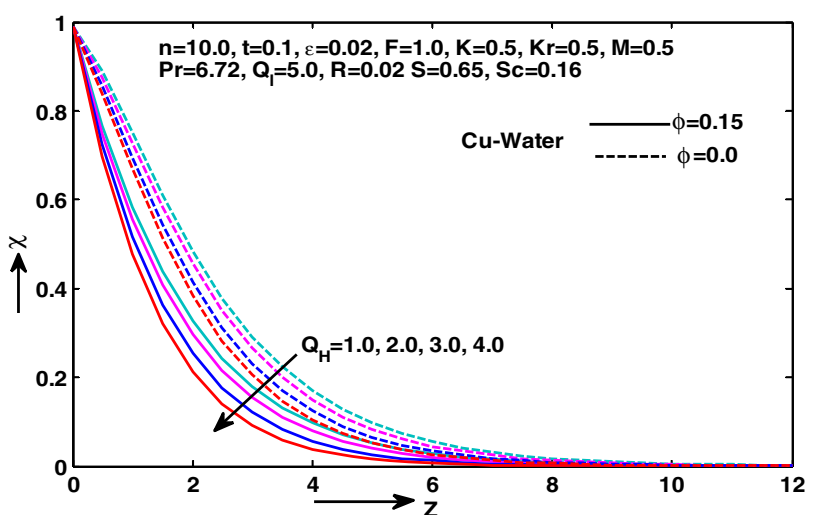

(a) Velocity for various values of $\mathrm{Q}_{\mathrm{H}}$

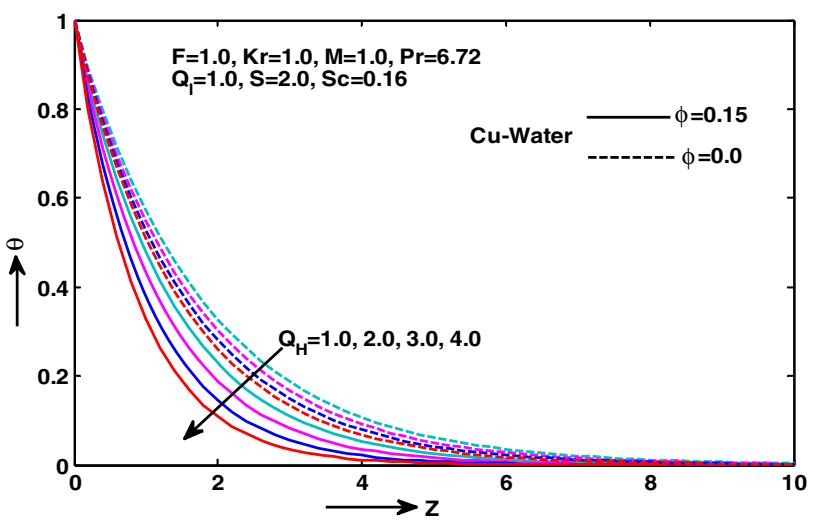

(b) Temperature for various values of $Q_{H}$

Fig. 6 a Velocity for various values of $Q_{H}$. b Temperature for various values of $Q_{H}$

respectively, for the $\mathrm{Cu}$ nanofluid particles $(\phi \neq 0)$ and water as the base fluid $(\phi=0)$ is illustrated in Fig. 4a, b. It is observed that an increase in $\mathrm{Kr}$ contributes to the decrease in the nanofluid velocity and temperature distributions. The effect of chemical reaction on the nanofluid velocity is less dominant in comparison to temperature profiles. These results are similar as noted in Satya Narayana et al. (2013a). Figure 5 presents typical profile for the nanofluid velocity profiles for different values of magnetic field parameter $M$. From the graph, it is obvious that the nanofluid velocity of the fluid decelerates with increase in the strength of magnetic field. The effects of a transverse magnetic field on an electrically conducting fluid give rise to a resistive-type force called the Lorentz force. This force has the tendency to slow down the motion of the fluid in the boundary layer. These results qualitatively agree with the expectations, since magnetic field exerts retarding force on the natural convection flow. In addition, it is seen that the nanofluid velocity is lower than for the pure water fluid. The effect of $\mathrm{M}$ on velocity profiles is reverse in case of Satya Narayana et al. (2013a). This is due to dominate role of nanofluid and radiation absorption. 


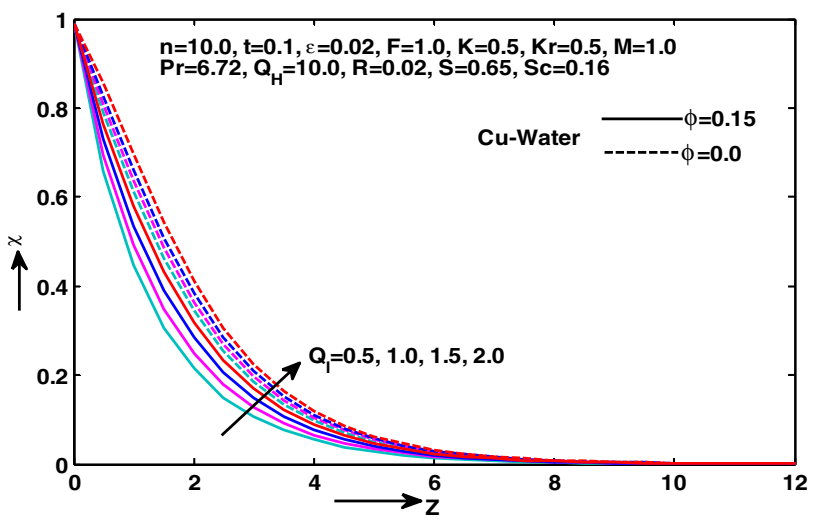

(a) Velocity for various values of $\mathrm{Q}_{1}$

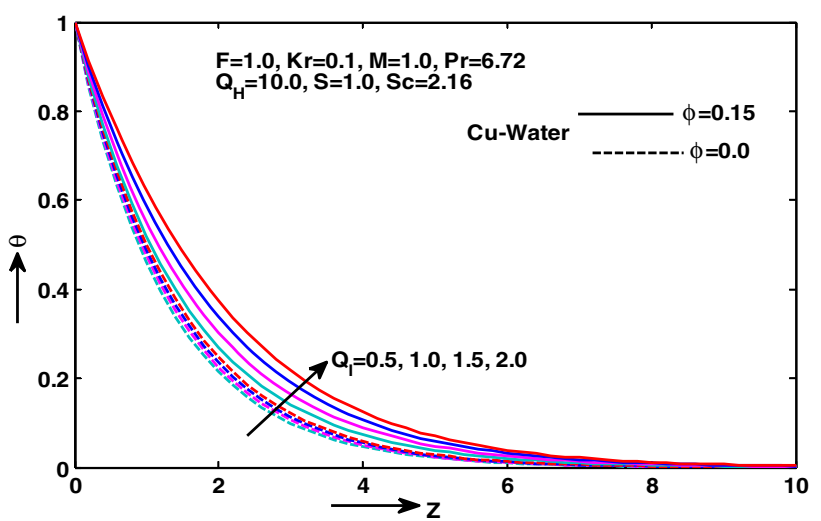

(b) Temperature for various values of $Q_{1}$

Fig. 7 a Velocity for various values of $Q_{1}$. b Temperature for various values of $Q_{1}$

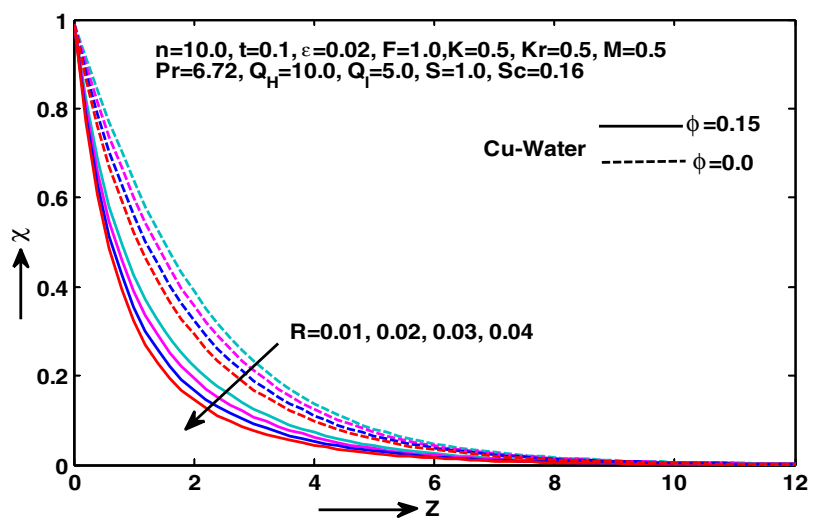

Fig. 8 Velocity for various values of $R$

Figure 6a, b, respectively, shows the nanofluid velocity and temperature profiles for different values of heat generation parameter $Q_{H}$. From the graphs, it is clear that there is a decrease in the nanofluid velocity and temperature profiles with an increasing in $Q_{H}$. This is due to the fact that when heat is absorbed, the buoyancy forces decrease

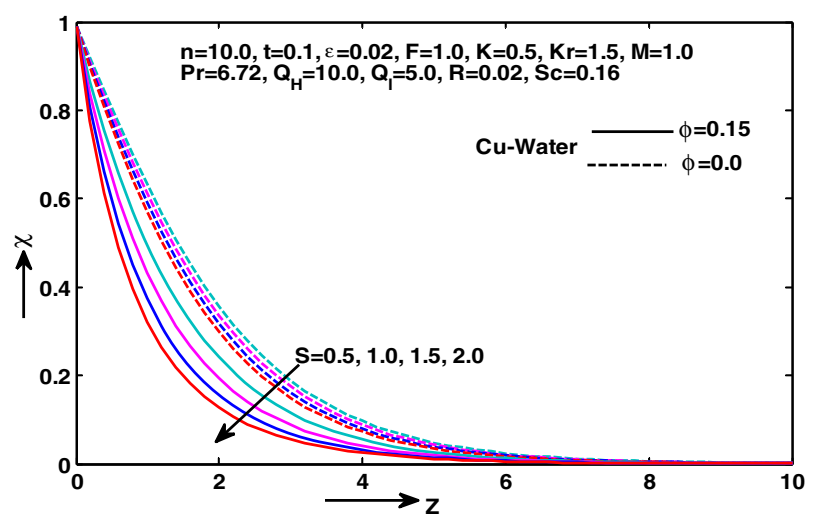

(a) Velocity for various values of $\mathrm{S}$

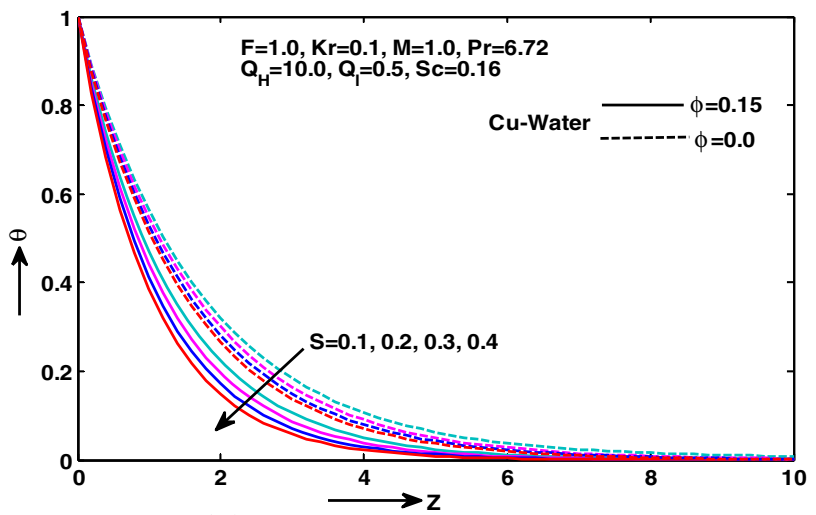

(b) Temperature for various values of $\mathrm{S}$

Fig. 9 a Velocity for various values of $S$. b Temperature for various values of $S$

which retard the flow rate and thereby give rise to a decrease in the velocity and temperature profiles. For various values of $Q_{1}$, the nanofluid velocity and temperature profiles across the boundary layer are shown in Fig. 7a, b. It is clear from the graphs that all the nanofluid velocity and temperature profiles increases with increase of $Q_{1}$. This is due to the fact that when heat is absorbed, the buoyancy forces accelerates the flow.

The influence of rotation parameter $R$ on the nanofluid velocity profiles for different values of $\mathrm{Cu}$ nanoparticles is shown in Fig. 8. From the figure, we see that the nanofluid velocity distribution across the boundary layer decreases with an increase of R. Figure 9a, b illustrates the effect of the suction parameter $S$ on the velocity and temperature profiles, respectively. It can be seen that the nanofluid velocity and temperature profiles decrease with the increase of the suction parameter. This indicates the usual fact that the suction stabilizes the boundary growth. These consequences are obviously supported from the physical point of view.

Figure 10a, b respectively, shows the nanofluid velocity and temperature profiles for different values of Schmidt number Sc. It is noticed that the effect of increasing values of Sc results in a decreasing the nanofluid velocity and 


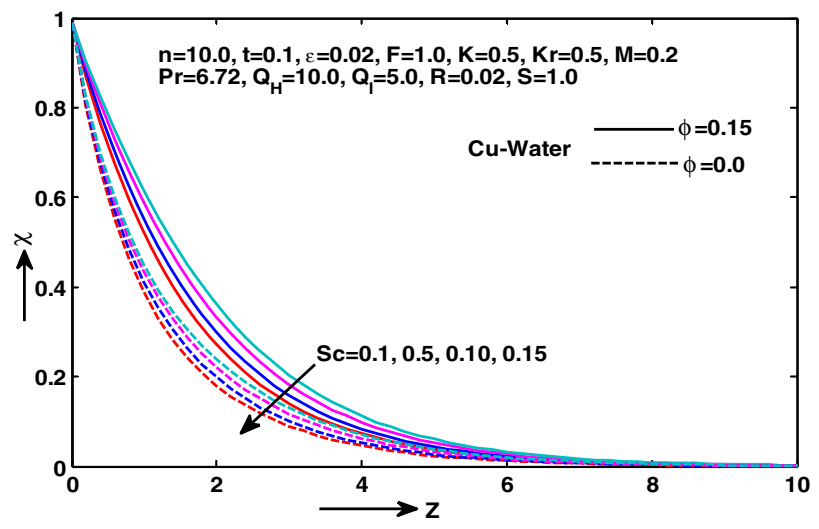

(a) Velocity for various values of Sc

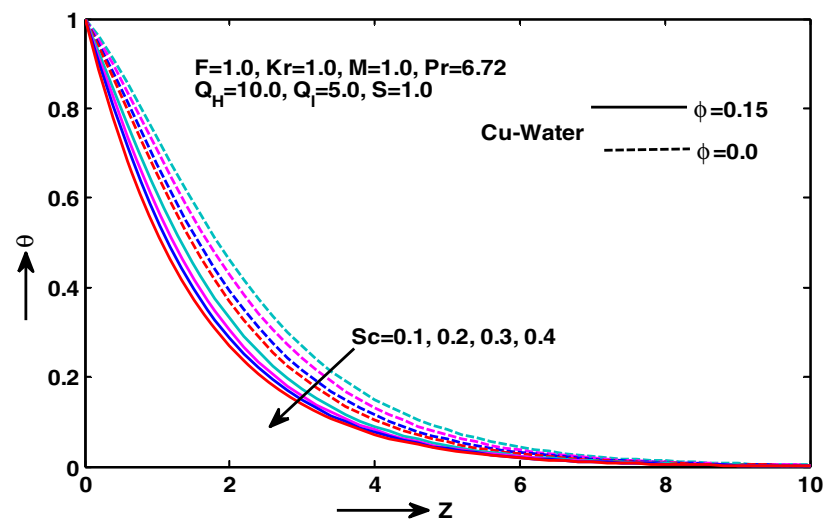

(b) Temperature for various values of $\mathrm{Sc}$

Fig. 10 a Velocity for various values of Sc. b Temperature for various values of $\mathrm{Sc}$

temperature distributions. Furthermore, the results show that the magnitude of $\mathrm{Sc}$ is less pronounced in case of nanofluids as compared to the base fluid. Figure 11a, b, respectively, illustrates the effect of the nanoparticle volume fraction $\phi$ on the velocity and temperature profiles. It is clear that as the nanoparticle volume fraction increases, the nanofluid velocity decreases and temperature distributions increases. This agrees with the physical behavior that, when the volume fraction of copper increases, the thermal conductivity increases, and then the thermal boundary-layer thickness increases.

Figure 12 illustrates the variations of the skin-friction coefficient with respect to the rotational parameter $R$. The skin-friction coefficient increases monotonically with increasing values of $R$. The effect of heat source on the rate of heat transfer is discussed in Fig. 13. It is observed that the rate of heat transfer of the fluid increases with the increase of heat source parameter. Increasing heat source has tendency to accelerate the thermal state of the fluid. Here, we compared our results with those of Satya Narayana et al. (2013a) for the micropolar fluid case. It is also worth noting that the nanofluid heat transfer rate is higher than that of the micropolar fluid. These results are clearly supported from the physical point of view.

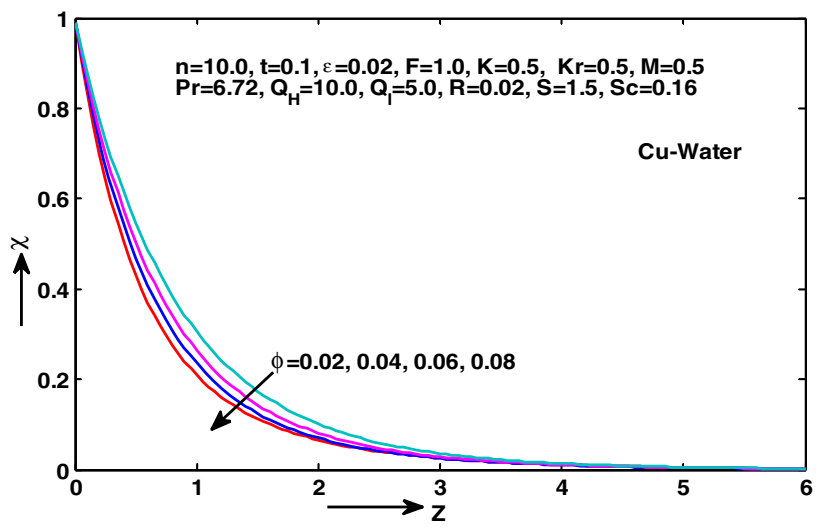

(a) Velocity for various values of $\phi$

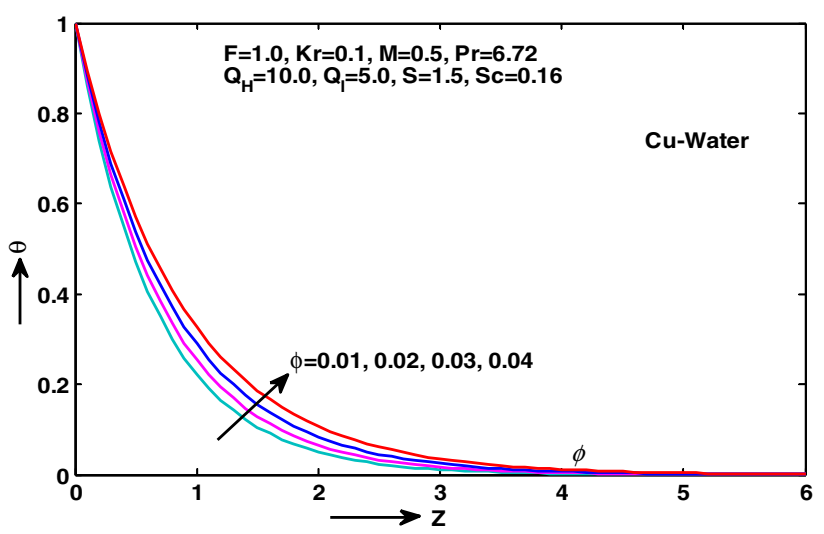

(b) Temperature for various values of $\phi$

Fig. 11 a Velocity for various values of $\phi$. b Temperature for various values of $\phi$

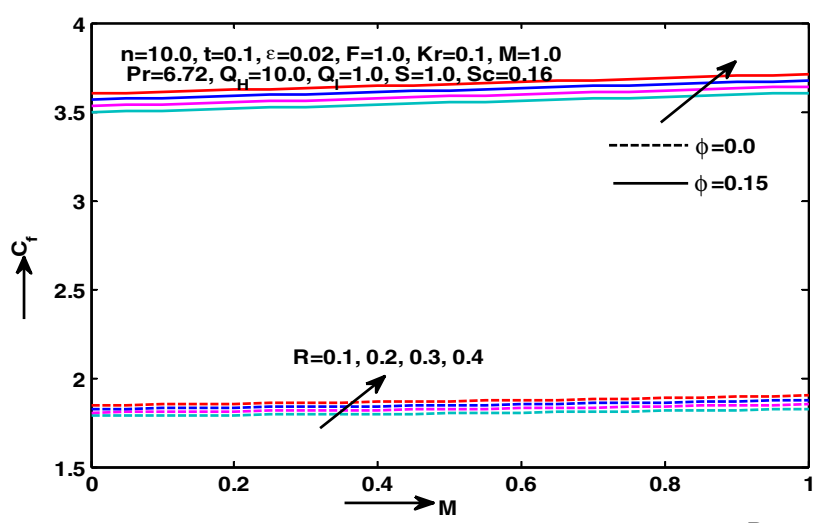

(a) Skinfricion coefficient for various values of $R$

Fig. 12 a Skin-friction coefficient for various values of $R$

Table 1 Thermo-physical properties of water and nanoparticles [Oztop and Abu-Nada (2008)]

\begin{tabular}{lllll}
\hline Physical properties & Water & $\mathrm{Cu}$ & $\mathrm{Al}_{2} \mathrm{O}_{3}$ & $\mathrm{TiO}_{2}$ \\
\hline $\mathrm{Cp}(\mathrm{J} / \mathrm{kg} \mathrm{K})$ & 4179 & 385 & 765 & 686.2 \\
$\rho\left(\mathrm{kg} / \mathrm{m}^{3}\right)$ & 997.1 & 8,933 & 3,970 & 4,250 \\
$K(\mathrm{~W} / \mathrm{m} \mathrm{K})$ & 0.613 & 400 & 40 & 8.9538 \\
$\beta \times 10^{-5}(1 / \mathrm{K})$ & 21 & 1.67 & 0.85 & 0.9 \\
\hline
\end{tabular}


Table 2 Comparison of skin-friction and Nusselt number of the present case with those of Satya Narayana et al. (2013b) and Hamad and Pop (2011) for $F=0.0, K=\infty, \mathrm{Kr}=0.0, \mathrm{Sc}=0.0, Q_{1}=0.0$

\begin{tabular}{|c|c|c|c|c|c|c|}
\hline \multirow[t]{2}{*}{$\operatorname{Pr}$} & \multicolumn{3}{|l|}{ Skin friction } & \multicolumn{3}{|c|}{ Nusselt number } \\
\hline & $\begin{array}{l}\text { Hamad and } \\
\text { Pop (2011) }\end{array}$ & $\begin{array}{l}\text { Satyanarayana } \\
\text { et al. (2013b) }\end{array}$ & Present study & $\begin{array}{l}\text { Hamad and } \\
\text { Pop (2011) }\end{array}$ & $\begin{array}{l}\text { Satyanarayana } \\
\text { et al. (2013b) }\end{array}$ & Present study \\
\hline 0.5 & 2.320 & 2.319970 & 2.31699069 & 5.967 & 5.967359 & 5.05226133 \\
\hline 1.0 & 2.258 & 2.257750 & 2.25755892 & 6.046 & 6.046127 & 6.04712801 \\
\hline 1.5 & 2.196 & 2.196289 & 2.19720830 & 6.125 & 6.125876 & 6.12582032 \\
\hline 2.0 & 2.134 & 2.134608 & 2.13460632 & 6.206 & 6.206602 & 6.20662363 \\
\hline
\end{tabular}

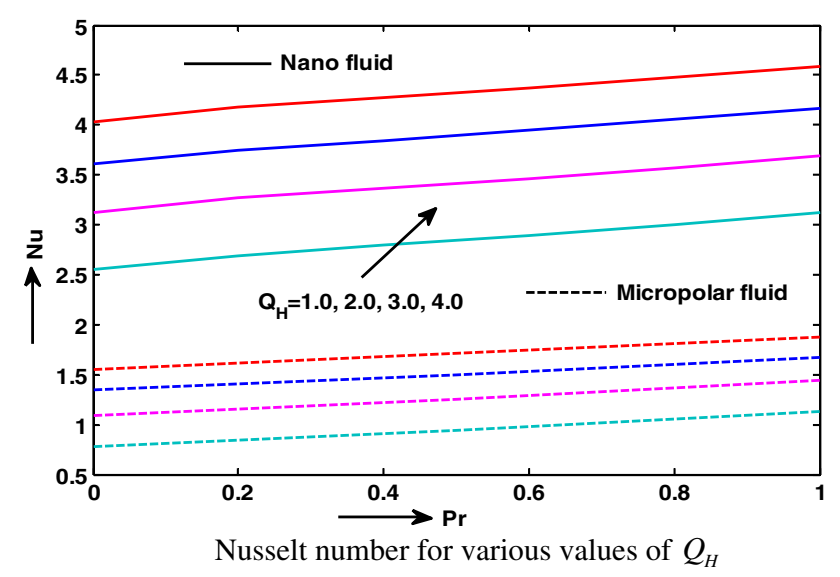

Fig. 13 Nusselt number for various values of $Q_{H}$

\section{Conclusions}

In this work, we have theoretically studied the effect of chemical reaction and radiation absorption on unsteady MHD free convection heat and mass transfer flow of an incompressible, nanofluid along a semi-infinite vertical flat plate in a rotating frame of reference. The governing equations are solved analytically by using perturbation technique. The effects of various parameters on velocity, temperature, skin friction and rate of heat transfer profiles are discussed through graphs and table. The following conclusions cab be made from the present investigation.

1. Increase in magnetic field decreases the momentum boundary layer for both base and nanofluid. It is observed that the velocity of a nanofluid is reduced extremely as compared to the base fluid. Magnetic parameter $M$ acts as a controlling parameter to control the flow fluid.

2. It is observed that the copper nanoparticles proved to have the maximum cooling performance for this porous vertical plate problem. This is due to the high thermal conductivity of $\mathrm{Cu}$. Thus, they can be used effectively for controlling/simulating the heat transfer rates.

3. The nanofluid motion and temperature profiles are retarded due to chemical reaction. The consumption of chemical species causes a fall in the concentration field which in turn diminishes the buoyancy effects due to concentration gradients. Hence the flow field is retarded.

4. The nanofluid velocity and temperature profiles increase with an increasing values of $Q_{l}$.

5. The skin-friction coefficient increases due to increase in magnetic parameter $(\mathrm{M})$ and rotation parameter (R).

6. The analytical results obtained in this work are more generalized form of Satya Narayana et al. (2013b) and Hamad and Pop (2011) and can be taken as a limiting case by taking $K \rightarrow 0 \mathrm{Kr} \rightarrow 0, F \rightarrow 0$ and $Q_{l} \rightarrow 0$.

7. It is worth noting that the values of rate of heat transfer in case of nanofluid are more pronounced than that of a micropolar fluid [Satya Narayana et al. (2013a)].

Open Access This article is distributed under the terms of the Creative Commons Attribution License which permits any use, distribution, and reproduction in any medium, provided the original author(s) and the source are credited.

\section{Appendix}

$A_{1}=(1-\phi)^{2.5} \quad A_{2}=\frac{k_{\mathrm{nf}}}{k_{f}}+\frac{4 F}{3}$
$A_{3}=1-\phi+\phi\left(\rho_{s} / \rho_{f}\right)$
$A_{4}=1-\phi+\phi(\rho \beta)_{s} /(\rho \beta)_{f}$
$A_{5}=1-\phi+\phi(\rho C p)_{s} /(\rho C p)_{f}$
$m_{1}=\frac{S S c+\sqrt{(S S c)^{2}+4 \mathrm{Kr} S c}}{2}$
$m_{2}=\frac{A_{1} A_{3} S+\sqrt{\left(\left(A_{5} / A_{2}\right) S \operatorname{Pr}\right)^{2}+4 Q_{H} / A_{2}}}{2}$
$m_{3}=\frac{A_{1} A_{3} S+\sqrt{\left(A_{1} A_{3} S\right)^{2}+4\left\{i R A_{1} A_{3}+(M+1 / K) A_{1}\right\}}}{2}$
$m_{4}=\frac{A_{1} A_{3} S+\sqrt{\left(A_{1} A_{3} S\right)^{2}+4\left\{i(R+n) A_{1} A_{3}+(M+1 / K) A_{1}\right\}}}{2}$
$m_{5}=\frac{A_{1} A_{3} S+\sqrt{\left(A_{1} A_{3} S\right)^{2}-4\left\{i(R-n) A_{1} A_{3}-(M+1 / K) A_{1}\right\}}}{2}$ 


$$
\begin{aligned}
B_{1} & =\frac{-\operatorname{Pr} Q_{l} A_{5}}{m_{1}^{2} A_{2}-S \operatorname{Pr} m_{1} A_{5}-Q_{H}} \quad B_{2}=1-B_{1} \\
B_{3} & =\frac{-A_{1} A_{4} B_{1}}{m_{1}^{2}-S m_{1} A_{1} A_{3}-\left\{i R A_{1} A_{3}+(M+1 / K) A_{1}\right\}} \\
B_{4} & =\frac{-A_{1} A_{4} B_{2}}{m_{2}^{2}-S m_{2} A_{1} A_{3}-\left\{i R A_{1} A_{3}+(M+1 / K) A_{1}\right\}} \\
B_{5} & =1-B_{3}-B_{4}
\end{aligned}
$$

\section{References}

Abdul-Kahar Rosmila, Kandasamy R, Muhaimin (2011) Scaling group transformation for boundary-layer flow of a nanofluid past a porous vertical stretching surface in the presence of chemical reaction with heat radiation. Comput Fluids 52:15-21

Abu-Nada E (2008) Application of nanofluids for heat transfer enhancement of separated flow encountered in a backward facing step. Int J Heat Fluid Flow 29:242-249

Abu-Nada E, Oztop HF (2009) Effect of inclination angle on natural convection in enclosures filled with $\mathrm{Cu}$-water nanofluid. Int $\mathrm{J}$ Heat Fluid Flow 30:669-678

Ahmad S, Rohni AM, Pop I (2011) Blasius and Sakiadis problems in nanofluids. Acta Mech 218:195-204

Alsaedi, Awais M, Hayat T (2012) Effect of heat generation/ absorption on stagnation point flow of nanofluid over a surface with convective boundary conditions. Commun Nonlin Sci Number Simulat 17:4210-4223

Bachok N, Ishak A, Nazar R, Pop I (2010a) Flow and heat transfer at a general three-dimensional stagnation point flow in a nanofluid. Phys B 405:4914-4918

Bachok N, Ishak A, Pop I (2010b) Boundary layer flow of nanofluid over a moving surface in a flowing fluid. Int J Therm Sci 49:1663-1668

Bachok N, Ishak A, Pop I (2011) Flow and heat transfer over a rotating porous disk in a nanofluid. Phys B 406:1767-1772

Brewster MQ (1972) Thermal radiative transfer properties. Wiley, New York

Buongiorno J (2006) Convective transport in nanofluids. ASME J heat Transf 128:240-250

Chamkha AJ, Gorla RSR, Ghodeswar K (2011) Non-similar solution for natural convective boundary layer flow over a sphere embedded in a porous medium saturated with a nanofluid. Transp Porous Media 86:13-22

Chamkha AJ, Abbasbandy S, Rashad AM, Vajravelu K (2012) Radiation effects on mixed convection over a wedge embedded in a porous medium filled with a nanofluid. Transp Porous Med 91:261-279

Choi S (1995) Enhancing thermal conductivity of fluids with nanoparticles, In: Siginer DA, Wang HP (eds.) Developments and applications of non-newtonian flows, FED-231, MD-66, ASME 99-105

Choi SUS, Zhang ZG, Yu W, Lockwood FE, Grulke EA (2001) Anomalously thermal conductivity enhancement in nanotubes suspensions. Appl Phys Lett 79:2252-2254

Ellahi R (2013) The effects of MHD and temperature dependent viscosity on the flow of non-Newtonian nanofluid in a pipe: analytical solutions. Appl Math Model 37(3):1451-1457

Ellahi R, Aziz S, Zeeshan A (2013) Non Newtonian nanofluids flow through a porous medium between two coaxial cylinders with heat transfer and variable viscosity. J Porous Media 16(3):205-216

Ganapathy R (1994) A note on oscillatory Couette flow in a rotating system. ASME J Appl Mech 61:208-209

Hady FM, Ibrahim FS, Abdel-Gaied SM, Mohamed Eid R (2012) Radiation effect on viscous flow of a nanofluid and heat transfer over a nonlinearly stretching sheet. Nanoscale Res Lett 7:229
Hamad AMM, Pop I (2011) Unsteady MHD free convection flow past a vertical permeable flat plate in a rotating frame of reference with constant heat source in a nanofluid. Heat Mass Transf. doi:10.1007/s00231-011-0816-6

Kakac S, Pramuanjaroenkij A (2009) Review of convective heat transfer enhancement with nanofluids. Int $\mathrm{J}$ Heat Mass Transf 52:3187-3196

Kameswaran PK, Narayana M, Sibanda P, Murthy PVSN (2012) Hydromagnetic nanofluid flow due to a stretching or shrinking sheet with viscous dissipation and chemical reaction effects. Int $\mathbf{J}$ Heat Mass Transf 55:7587-7595

Khanafer K, Vafai K, Lightstone M (2003) Buoyancy driven heat transfer enhancement in a two dimensional enclosure utilizing nanofluids. Int J Heat Mass Transf 46:3639-3653

Makinde OD, Aziz A (2011) Boundary layer flow of a nanofluid past a stretching sheet with a convective boundary condition. Int $\mathbf{J}$ Therm Sci 50:1326-1332

Hajipour M, Molaei Dehkordi A (2012) Analysis of nanofluid heat transfer in parallel-plate vertical channels partially filled with porous medium. Int $\mathrm{J}$ Therm Sci 55:103-113

Mazumder BS (1991) An exact solution of oscillatory Couette flow in a rotating system. ASME J Appl Mech 58(4):1104-1107

Muthtamilselvan M, Kandaswamy P, Lee J (2010) Heat transfer enhancement of Copper-water nanofluids in a lid-driven enclosure. Comm Nonlin Sci Numer Simulat 15:1501-1510

Narahari M, Akilu S, Jaafar A (2013) Free convection flow of a nanofluid past an isothermal inclined plate. Appl Mech Mater 390:129-133

Nield DA, Kuznetsov AV (2009) The Cheng-Minkowycz problem for natural convective boundary-layer flow in a porous medium saturated by a nanofluid. Int J Heat Mass Transf 52:5792-5795

Olanrewaju PO, Olanrewaju MA, Adesanya AO (2012) Boundary layer flow of nanofluids over a moving surface in a flowing fluid in the presence of radiation. Int J Appl Sci Tech 2(1):274

Oztop HF, Abu-Nada E (2008) Numerical study of natural convection in partially heated rectangular enclosures filled with nanofluids. Int J Heat Mass Transf 29:1326-1336

Rana P, Bharava R (2012) Flow and heat transfer of a nanofluid over a nonlinearly stretching sheet. Commun Nonlin Sci Number Simulat 7:212-226

Satya Narayana PV, Venkateswarlu B, Venkataramana S (2013a) Effect of chemical reaction and thermal radiation on MHD micropolar fluid in rotating frame of reference with constant heat source. J Energy Heat Mass Transf 35(3):197-214

Satya Narayana PV, Venkateswarlu B, Venkataramana S (2013b) Thermal radiation and heat source effects on a MHD nanofluid past a vertical plate in a rotating system with porous medium. Heat Transf Asian Res. doi:10.1002/htj.21101

Satya Narayana PV, Venkateswarlu B, Venkataramana S (2013c) Effects of hall current and radiation absorption on MHD micropolar fluid in a rotating system. Ain Shams Eng J 4(4):843-854

Tiwari RK, Das MK (2007) Heat transfer augmentation in a two-sided lid-driven differentially heated square cavity utilizing nanofluids. Int J Heat Mass Transf 50:2002-2018

Uddin Z, Harmand S (2013) Natural convection heat transfer of nanofluids along a vertical plate embedded in porous medium. Nanoscale Res Lett 8:64

Umavathi JC (2013) Effect of thermal modulation on the onset of convection in a porous medium layer saturated by a nanofluid. Transp Porous Med 98:59-79

Yu DM, Routbort JL, Choi S (2008) Review and comparison of nanofluid thermal conductivity and heat transfer enhancements. Heat Transf Eng 29:432-460

Zheng L, Zhang C, Zhang X, Zhang J (2013) Flow and radiation heat transfer of a nanofluid over a stretching sheet with velocity slip and temperature jump in porous medium. J Franklin Inst. doi:10. 1016/j.jfranklin.2013.01.022 